RICERCHE SULLE SCARICHE INTERNE ED ESTERNE DEI CONDENSATORI; MEHORIA DEL, PROF. EMILIO VILLARI $\left.{ }^{*}\right)$.

Che realmente per la carica istantanea accorra nella bottiglia maggiore elettricità dopo averla scaricata rapidamente che dopo averla scaricata lentamente può dimostrarsi direttamente. Ed infatti, con una batteria di 8 bottiglie di 32 unita caricavo istantaneamente, attraverso il galvanometro, una bottiglia con $21 \mathrm{~cm}$. di altezza di armaturo, e misuravo la corrente di carica, dopo che la bottiglia in una prima serie di esperienze era stata sempre scaricata istantaneamente, ed in una seconda sempre lentamente. Le misure furono così più volte eseguite ed i resultati ottenuti sono i seguenti:

TABELLa XII.

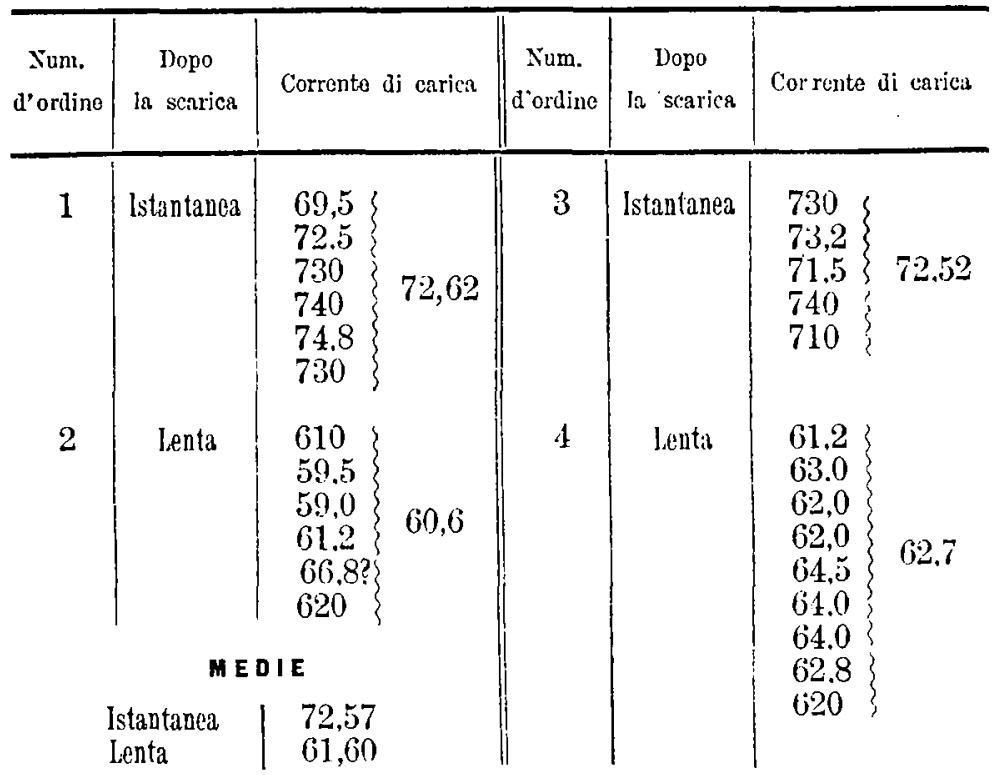

Onde si vede che la corrente di carica fu di $72^{\mathrm{mm}}, 6$. dopo la scarica istantanea, e di 61,6 dopo la scarica lenta ${ }^{2}$ ). Che poi

1) Continuazione e fine. Vedi pag. 143.

2) Le scariche furono ciasenna volta completate con circuito inetallico. 
la differenza sia realmente dovuta al distruggersi o no della zona diffusa e quindi alla antecedente scarica più o meno completa, può ancora dimostrarsi ripetendo le precedenti esperienze col caricare la bottiglia con la solita batteria, ma sempre lentamente per mezzo di curdoni bagnati attaccati ai serrafili $a, a^{\prime}$. Così 0 perando mancava la grando diffusione elettrica, ed allora per la carica passava sempre nella bottiglia, ad un dipresso, la stessa quantità di elettricità comunque fosse stata scaricata dianzi, cioè o rapidamente o lentamente. La Tabella seguente riporta $i$ risultati di tali esperimenti:

\section{ThBeLLa XIII.}

\begin{tabular}{|c|c|c|c|c|c|}
\hline \multicolumn{3}{|c|}{ 1. SERIE } & \multicolumn{3}{|c|}{ II. SERIE } \\
\hline $\begin{array}{c}\text { Dopo } \\
\text { Ia scarics }\end{array}$ & Deviazione & di carica & $\begin{array}{c}\text { Dopo } \\
\text { las scarica }\end{array}$ & Deviaziono & di carjea \\
\hline Rapida & $\begin{array}{l}58,5 \\
60,5 \\
59,8 \\
60,2 \\
57.0 \\
57,5 \\
57,8 \\
59,6\end{array}$ & 57.6 & Rapida & $\begin{array}{l}58,0 \\
59,5 \\
61,5 \\
60,4 \\
59,0 \\
61,9\end{array}$ & $\check{5} 9,9$ \\
\hline Lenta & $\left.\begin{array}{l}59,8 \\
60,4 \\
61,3 \\
60,2 \\
60,5 \\
58,5\end{array}\right\}$ & 60,4 & Irenta & $\begin{array}{l}58,0 \\
57,8 \\
58,7 \\
58,9 \\
59,5 \\
58,5\end{array}$ & 58,7 \\
\hline
\end{tabular}

\begin{tabular}{c|c} 
Dopo la scarica & Deviazioni di carica \\
Rapida & 58,75 \\
Lenta & 59,55
\end{tabular}

Onde si rileva che la bottiglia caricata lentamente e quindi scaricata, sia istantaneamente sia lentamente, ricevè dopo, per la nuova carica istantanea, sempre la stessa quantità di elettrico misurata da una deviazione di circa $59^{\mathrm{mm}}$. 
Da ultimo aggiungerd che arendo caricato, al solito, una bottiglia istantaneamente con una batteria di 8 bottiglie e 27 cariche, ed avendo quindi col galvanometro misurata la sua corrente di scarica promossa, sia fra due palline, sia fra punte e palline, trovai detta corrente essere affatto costante nei due casi, come mostra la Tabella seguente:

Tabeila XIV.

\begin{tabular}{c|c||c|c}
\hline \multicolumn{3}{c}{ DEVIAZION1 } & $\begin{array}{c}\text { PER } \\
\text { fra }\end{array}$ \\
\hline $\begin{array}{c}\text { Num. } \\
\text { d'ordine }\end{array}$ & Palline & Num. & SCARICA \\
\hline d'ordine & Punte o palline \\
\hline 2 & 47 & 1 & \\
4 & 47 & 3 & 46 \\
6 & 47 & 5 & 47 \\
8 & 48 & 7 & 47 \\
10 & 46 & 9 & 46 \\
12 & 44 & & $-\overline{46,4}$ \\
& medie 46,5 & &
\end{tabular}

\begin{tabular}{r|l||r|l}
\multicolumn{5}{c}{ Medesime Esperienze } \\
1 & 46 & 2 & 45 \\
3 & 43 & 4 & 45 \\
5 & 47 & 6 & 46,6 \\
7 & 45 & 8 & 48 \\
9 & 45 & 10 & 47 \\
11 & 47 & & 43 \\
13 & 47 & & 44 \\
15 & 47 & & 47 \\
17 & 45 & & \\
& mellie 45,55 & & $\overline{45,7}$
\end{tabular}

Onde se ne inferisce che in queste condizioni la quantità di elettricità che produce la corrente della scarica esterna non dipende, in modo sensibile, dalla maniera di scaricare il condensatore. 


\section{$\$$ III.}

Sul valore della scarica interna ed esterna dei condensatori.

Lsposi gia in altre Memorie i resultati relativi all' energia delle scariche, si all' esterno che all' interno di condensatori, ora dirò di alcuni nuovi fenomeni osservati intorno al medesimo soggetto.

Le ricerche relative alle scariche interne furono eseguite col mio termometro Leido-elettrico altra volta descritto ${ }^{1}$ ). Esso, quale ultimamente l'ho costruito, è disegnato nella figura 14, ed c̀ formato da una campana di vetro mnn' la quale ha il suo orlo iuferiore masticiato in una ghiera di ottone $m n^{\prime}$, che a vite si fissa al recipiento d'ottone $n n^{\prime}, c c^{\prime}$ così strettamento da tener l'aria. Nel vano della campana e del recipiente, whe costituisce il termometro, è contenuta una bottiglia di Leida cilindrica, alta $50 \mathrm{~cm}$. e larga $10 \mathrm{~cm}$. La sua armatura esterna, mercè tre forti molle $f, f$ comunica col recipiente d'ottone, $\theta$ l'interna del pari con tre forti molle $o, o^{\prime}$ è unitia al tubo d'ottone $t p$, e per esso alle palline $q$. Il tubo passa attraverso un tappo di sughero che chiude ermeticamente l'apertura del coperchio $m$ della campana, e poscia si continua cou un tubo di gomma ti e col cannello di vetro $i b$, contenente un indice di alcool, il quale serve a misurare le dilatazioni dell'aria del termometro. $L$ apparecchio è unito ad una base di legno $B B^{\prime}$ che fa da sostegno. La pallina $q$ serve per caricare o searicare la bottiglia con l' eccitatoro $E$. Perchè l' apparecchio funzioni regolarmente ò necessario che le sue chiusure sieno perfetto così da tenere l'aria, e che la campana $m n n$ ' sia verniciata internamente ed esternamente.

$\Lambda$ vanti tutto dirò come le frange di scarica che si formano ai bordi delle armature svolgono del calore, pel quale non solo l'aria ma anche il vetro ove esse si producono si riscaldir sensibilmente. Ed invero, se si carica o scarica più volte di seguito fortemente e con rapiditì la bottiglia nel precedente termometro si vedrì, che esso mostra una dilatazione persistente di circa $10^{\mathrm{mm}}$

1) Villari. Sulle scariche internc dei cundensatori; $l . c$. 
per ogni cento scariche: la quale sparisce lentamente, e dopo parecchi minuti. Se le stesse esperienze si fanno con una bottiglia fuori del termometro, e poscia se ne esamini, con una termopila, la faccia esterna del vetro disarmato si troverà riscaldata soltanto la regione prossima al bordo dell' armatura.

Cio premesso, le mie nuove indagini ebbero lo scopo di de. terminare l'energia delle scariche interne delle bottiglie nel momento della loro carica e scarica istantanea. Perciò caricavo la bottiglia del termometro rapidamente, riunendone le armature con quelle di una batteria di 4 delle consuete giare caricate con 14 unità; ed osserravo la dilatazione del termometro. Poscia scaricavo la bottiglia con un eccitatoro a palline, e del pari misuravo la dilatazione del termometro dovuta alla scarica interna ${ }^{1}$ ): ed i resultati che ottenni da varie di questo misure sono i se. guenti:

TABELla XV.

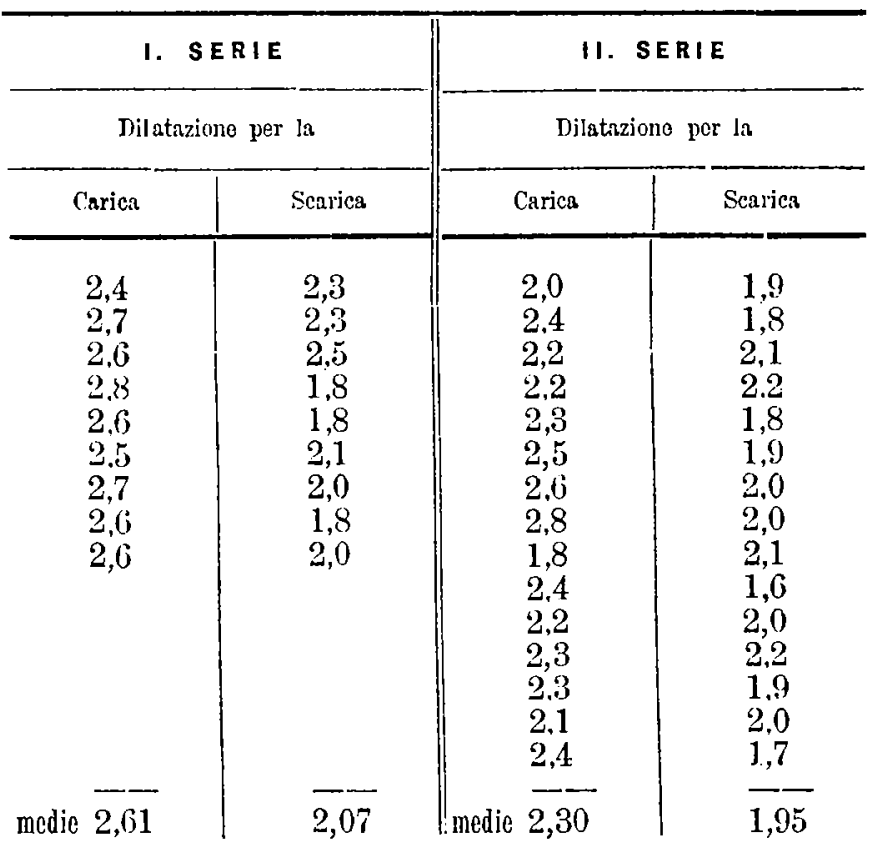

1) Le frange che si producono nelle cariche o scaricho interne della bottiglin, oltro - he dilatare direttamento l'aria la dilatano altresi pol calerico che esse svolgouo sul ve- 
I numeri precedenti sono fra loro assai concordi, e mostrano che la dilatazione termometrica o l'energia delle frange di carica è alquanto maggiore di quella delle frange di scarica, perchè con la carica si diffondo più elettricità sul vetro che non se ne neutralizza nella scarica: il che concorda sia con le osserrazioni galvanometriche sia con quelle eseguite mercè le figure elettriche dette di sopra.

Poscia cercai di determinare l'influenza che aveva la maniera di caricar la bottiglia sul valore della sua scarica interna. Percio adoperai come sorgente elettrica costante una batteria di 4 bottiglie, caricata con 14 unità, la cui armatura esterna era unita all'omonima della bottiglia del termometro Leido-elettrico; e per caricar la bottiglia riunivo la sua armatura interna con quella della batteria, talvolta direttamente con un eccitatore a palline (carica istantanea): ed altre volte con lo stesso eccitatore, ma prolungato con un cordone bagnato di una trentina di centimetri di lunghezza (scarica lenta): nel quale ultimo caso subito completavo la carica mercè comunicazioni metalliche. Dopo caricata la bottiglia nei due modi indicati la scaricaro istantaneamente con l'eccitatore a palline, e misuravo vei due casi la dilatazione del termometro dovuta alla scarica interna. La batteria avanti ogni nuova esperienza veniva sempre scaricata con l'eccitatore metallico. I risultali di questi confronti sono qui di seguito riportati: 
T'abelia XVi.

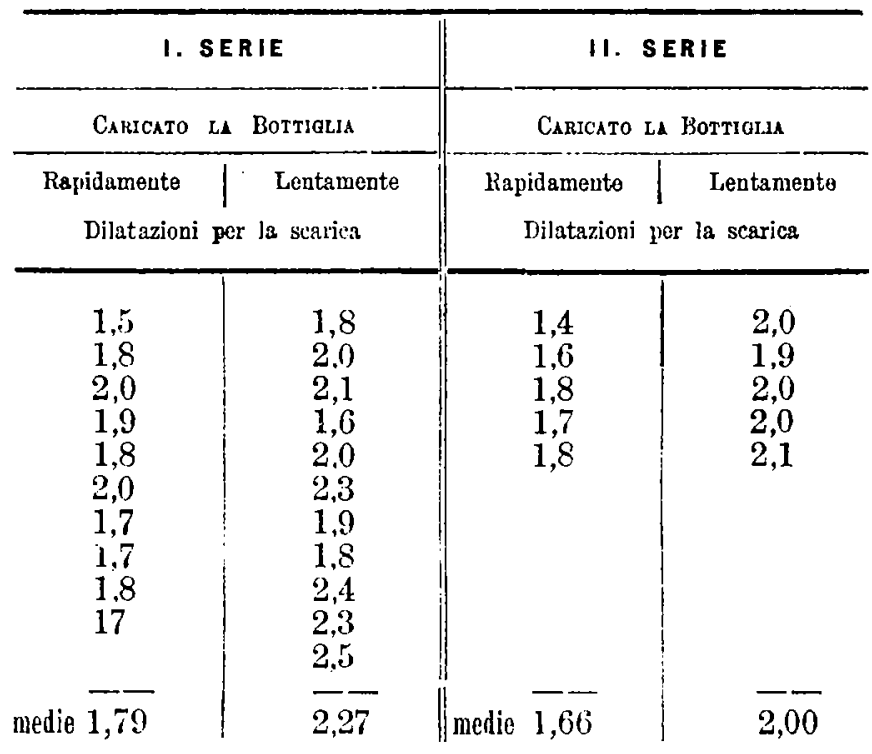

Tutti i numeri precedenti mostrano che la intensità della scarica interna è minore quando la bottiglia fu caricata lentamente, ed è più cospicua se fu caricata istantaneamente: e la differenza è sicura perchè sensibile e perchè confermata da molte esperienze tutte concordi. Codesta differenza è al solito dovita alla maggiore diffusione elettrica prodotta dalla carica istantanea relativamente alla lenta, e quindi al maggiore riflusso elettrico che si verifica per la scarica successiva alla carica istantanea.

Dopo le precedenti ricerche feci alcune poche esperienze per determinare la relazione fra il valore della scarica interna ed esterna del condensatore, riservandomi di completare in seguito queste prime ricerche.

Ed avanti tutto ricorderò come io abbia di già dimostrato in altra occasione ${ }^{1}$ ) che la scarica interna di una bottiglia non varia, se s' aumenta la sua armatura interna: ed invece s' accresce assai sensibilmente quando la detta armatura si faccia più piccola dell' esterna. Questi fenomeni sono dovuti alla spiccata

1) Villari. Sulle scariche intorne dei condensatori; I. e. 
200

azione che esercita sulle scariche interne il potenziale della bottiglia.

Quando in un condensatore ad armature eguali si aumenta una di esse, il potenziale di una data massa elettrica non varia sensibilmente e perciò non raria neanche la scarica interua. Se per lo contrario si diminuisce l'estensione di una delle due armature eguali, s'accresce allora il potenziale della data massa elettrica ${ }^{1}$ ) ed in conseguenza s' accresco la scarica interna.

F partendo da queste considerazioni studiai l'influenza che esercita la estensione delle armature sul valore della scarica esterna: perciò sperimentai con una delle consuete giare, che caricaro internamente e sempre con 3 unitia; quindi la scaricavo per mezzo del mio termometro eccitatore, o misuravo le dilatazioni in esso prodotte dalla scintilla eccitatrice unica, ossia dalla scarica esterna. Tale giara in alcune esperienze aveva le armature eguali e di $38 \mathrm{~cm}$. di altezza ciascuna: in altre fu accorciata l' interna e ridotta a $30 \mathrm{~cm} .{ }^{2}$ ); ed i risultati ottenuti da molte unisure furono i seguenti:

1) Isa capacitś di un condensitore diponde pressochi esclusivamonte dell' armatura minore, o come suol dirsi, dall' estonsione armatir. Belli, Trattato di fisica, $\S 1033$ o sog.

2) La bottiglia areva lo armaturo di $30 \mathrm{~cm}$., che prolungai di $8 \mathrm{~cm}$, attaccando sul vetro due strisce di stagnola unte con un poco di sego: per scorciar poscia una delle armature se ne staccava $J_{a}$ striscia di stirgnola colrispondente. 
'Tabelia XVII.

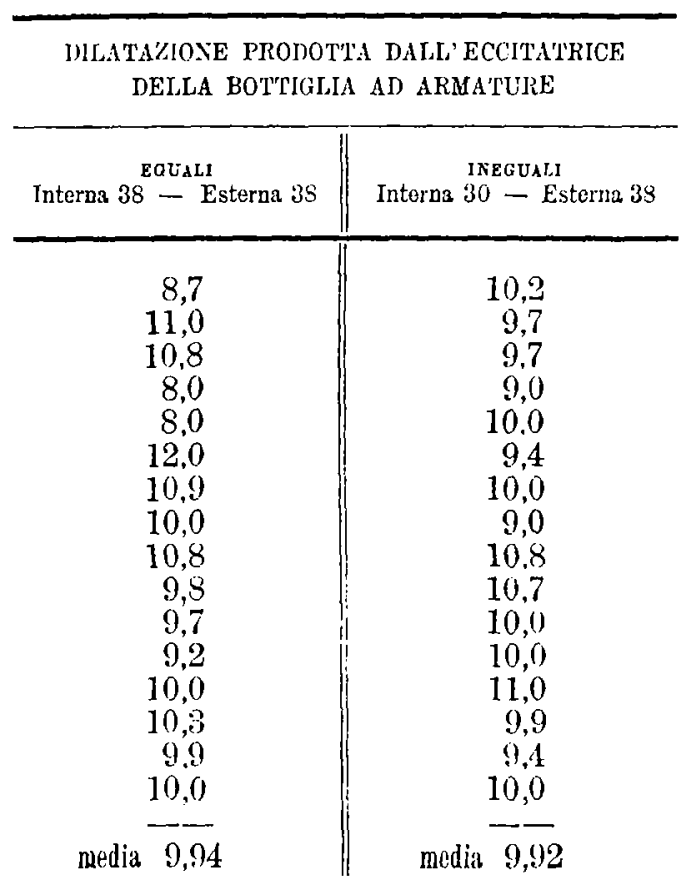

I numeri precedenti mostrano che la dilatazione prodotta dall' eccitatrice esterna fu costante, sia con la bottiglia ad armature eguali sia cou quella interna piì piccola dell' esterna: e perciò si conclude che l'aumento di potenzialo nel caso precedente è stato insufficiente a mostrare un aumento di energia nella scarica esterna.

Quindi rifeci le suaccennate misure caricando la stessa bottiglia con quattro uniti olettriche, invece dello tre adoperate precedentemente, ed ottenni i risultati qui di seguito riferiti : 
TABella XVIII.

\begin{tabular}{|c|c|}
\hline \multicolumn{2}{|c|}{$\begin{array}{l}\text { DILATAZIONE GENERATA DALL'ECCITATRICE } \\
\text { PRODOTIA DALLA BOTTIGLIA AD ARMATURA }\end{array}$} \\
\hline $\begin{array}{c}\text { EGUALI } \\
\text { Interna } 38 \text { - Esterna } 38\end{array}$ & $\begin{array}{ll} & \text { INEGUALI } \\
\text { Interna } & 30-\text { Esterna } 38\end{array}$ \\
\hline 13,9 & 18,0 \\
\hline 17,0 & 26,0 \\
\hline 16,8 & 18,9 \\
\hline 17,0 & 27,0 \\
\hline 18,0 & 25,0 \\
\hline 18,0 & 18.5 \\
\hline 17,9 & 29.0 \\
\hline 18,8 & 20,6 \\
\hline 17,0 & 19,4 \\
\hline 19,5 & 18,6 \\
\hline 15,0 & 19,8 \\
\hline 16.0 & 23,5 \\
\hline 17,5 & 20.0 \\
\hline 15,0 & 20,8 \\
\hline 18,0 & 18,7 \\
\hline 16,5 & 22,0 \\
\hline media $\overline{16,99}$ & media $\overline{21,61}$ \\
\hline
\end{tabular}

Questi numeri mostrano chiaro che la dilatazione prodotta dall' eccitatrice esterna, generata dalla scarica di una bottiglia ad armature eguali, è sensibilmente minore di quella prodotta dalla medesima dopo però che l'armatura interna sia stata scorciata di $8 \mathrm{~cm}$. E ciò è doruto all' aumento di potenziale, il quale oltre dell' aumento della scarica esterna è accompagnato, come è noto, da un aumento della scarica interna.

Laonde concludendo potremo dire che diminuendo una delle armature di una bottiglia ad armature eguali, il potenziale di una data sua carica s'accresce, ed in conseguenza il valore della scarica aumenta, sì nell' interno che nell' esterno di essa.

Riassunto. - Nella carica di un condensatore l' elettricità delle armature si diffonde parzialmente sul vetro circostante, e da questo ritorna in parte a quelle, nel momento della scarica: cosicchè produconsi come un flusso e riflusso elettrico, i quali 
studiati con un galvanometro a specchio ed a perfetto isolamento, condussero al risultato seguente:

Quando in un condensatore in equilibrio elettrico, una delle armature perde elettricitì (nel concetto unitario), il vetro circostante supplisce in parte tali perdite, e produconsi correnti dirette verso l'armatura. Se, per lo contrario, una armatura acquista elettricità una parte di essa si diffonde sul vetro vicino, producendo correnti dirette verso il vetro. F tali flussi elettrici, quando sono sufficientemente energici, danno luogo alle frange di carica e di scarica.

Un condensatore, per mezzo di una batteria elettrizzata può caricarsi rapidamente o lentamente; e poscia può del pari scaricarsi lentamente o rapidamente. Le correnti che produconsi sul vetro ai bordi delle armature, sono più energiche nel momento della carica che della scarica; ed altresì piì energiche per la carica e scarica istantanea che per la lenta.

Costrutto un secondo condensatore sul vetro libero d'una bottiglia, o distante dalle armature di questa per circa 30 millin. si producono grosse e vigoroso scintille fra i due condensatori nel momento della carica e scarica istantanea della bottiglia. Se pero si riuniscono le armature interne dei due condensatori mercè una di stagnola, allora nel momento della carica e scarica istantanea non si manifestano, come è naturale, più scintille internamente: e del pari una sola se ne produce esternamente, la quale scorre in prossimita della lista di stagnola interna, quasi fosse attratta da questa, sebbene essa sia percorsa da scarica di direzione opposta a quella della scintilla.

Quando si carica istantaneamente un condensatore, dalle armature si diffonde sul vetro molta maggior copia di elettricità, che nel caso della carica lenta: $\theta$ parimente per la scarica istantanea, molto maggiore e la copia della elettricità diffusa che ricorre alle armature, che per la scarica lenta. Laonde un condensatore che si carica e scarica istantaneamente, può considerarsi come più ampio, e percio come più capaco della sua parte armata, onde si constatano i seguonti fenomeni col galvanometro.

In un condensatore caricato lentamente poca elettricita suolsi diffondere sul vetro, perciò la sua scarica, sia lenta sia ra- 
204

pida. produce sensibilmente la stessa devinzione del galvanojnetro ${ }^{1}$ ).

Per la carica istantanea passa in un condensatore piu elettricitì che per la carica lenta, quando si abbia l'arvertenza di scaricarlo ciascuna volta istantineamente, a fine di distruggere la carica diffusa antecedente.

La scarica istantanea consta di più clettricita, quando il condensatore fu caricato istantaneamente, che quando lo fu lentamente.

In una bottiglia, che si carichi istantaneamente accorrerà più elettricità, se la sua carica avvenga dopo arcrla scaricata rapidamente che dopo scaricata lentamente.

In una bottiglia che si carichi lentamente, con una data batteria, accorre sempre l'istessa quantiti di elettricitì sia dopo averla scaricata lentamente sia istantaneamente, per la poca diffusione che vi produce la carica lenta.

Introducendo una bottiglia in un termometro ad aria si osserva che le dilatazioni di questo. prodotte per lo frange di carica, sono più cospicue di quelle prodotte per le frange di scarica, perchè magogiore è il flusso elettrico nel primo che nel socondo caso.

Le frange della scarica istantanea d' una bottigrlia, sono più energiche e generano maggiore dilatazione nel termometro se la bottiglia fu caricata istantaneamente che se lo fu lentamente.

L' anpiezza delle dilatazioni del termometro, o l'energia delle scariche interne non raria, se si accresce l' estensione di una delle armature eguali della bottiglia, non modificandosene con ciò sensibilmente il potenziale di una data carica. Inveco se una dello due armature eguali si scarica, la capacità del condensatore decresce; il potenziale d' una data carič cresce, e con essa aumenta la energia della scarica, o tale aumento si scorge sì nella scarica esterna che nell'interna della boltiglia, quando si sperimenti con elevate cariche elettriche.

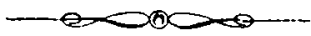

1) In quoste esperienze la bottiglia si soleva caricilro istantaneamente o lentamente riuuendo le sile aruature con quelle di una batteria gia carica, per mezzo di un eccitatore o tutto metallico ovvero prolungato con un cordone bagnato: e nel medesimo modo si producevano lo scariche istantance o lente. F a notarsi cho le caricho o scariche lente subito si completavano cliudendo il circuito metalicamente. 